\title{
Characterization of supersonic low pressure plasma jets with electrostatic probes
}

\author{
M Gindrat $\uparrow$, J-L Dorier $\uparrow$, Ch Hollenstein $\dagger$, \\ A Refke $\ddagger$ and G Barbezat $\ddagger$ \\ † Swiss Federal Institute of Technology, Plasma Physics Research Centre, CH-1015 \\ Lausanne \\ $\ddagger$ Sulzer Metco AG, Rigackerstr.16, CH-5610 Wohlen \\ E-mail: malko.gindrat@epfl.ch
}

\begin{abstract}
.
Measurement of key plasma jet properties in a low pressure environment, such as the Mach number, electron density and temperature, were performed using double Langmuir probes and Mach probes. In particular, under-expanded jets are studied in detail by performing complete mappings of plasma jet properties at 10 and 2 mbar chamber pressure. These results show that the measured physical properties are consistent with the jet flow phenomenology such as the presence of periodic expansion and compression zones, the effect of the pressure and the location of the shocks. It is shown in particular that for a highly under-expanded jet at 2 mbar, the Mach number reaches 2.8 in the first expansion zone followed by a strong drop to subsonic flow revealing the presence of a Mach reflection. The flow is accelerated further and a periodic structure of compression/expansion cells is observed until the local static pressure is in equilibrium with the surrounding pressure. These results contribute to the understanding of the supersonic plasma jet behaviour at low pressure and can be used to quantify the deviation from LTE. The extensive mapping of the measured physical properties of the jet will also serve as input for modelling.
\end{abstract}

Submitted to: Plasma Sources Sci. Technol. 


\section{Introduction}

Low Pressure Plasma Spraying (LPPS) processes use a DC plasma jet expanding at low pressure for fast deposition of dense coatings in a controlled atmosphere. The LPPS technology is widely used industrially in particular in the aeronautics and medical industries among others [1]. Unlike atmospheric pressure plasma jets, which have been extensively studied experimentally and theoretically, the growth of interest in low pressure DC plasma jets only occurred recently. However, the process development has been mainly based on empirical methods and the basics of the physical mechanisms that govern them still remain to be investigated. Further improvement of the processes requires, in particular, the knowledge of physical properties of the plasma jet such as the temperature, flow velocity and plasma density [2]. Low pressure plasma jets present unconventional properties such as low collisionality, large dimensions and supersonic flow. Therefore specific diagnostics have to be adapted to these conditions.

A diagnostic extensively used in plasma spraying is optical emission spectroscopy (OES) which is non-intrusive and gives information about the plasma species. However, determination of the temperature, for example, which is obtained using the Boltzmann plot method, relies on the assumption of local thermodynamic equilibrium (LTE), which is no longer satisfied at lower working pressure. The result of the deviation from LTE is that the heavy particle, electron and excitation temperatures are different. Therefore, different interpretations of OES measurements in plasma jets at reduced pressure have been developed and are mainly used to evaluate the deviation from the LTE as a function of the working pressure $[3,4,5]$.

Another diagnostic tool, the enthalpy probe system, has been used in compressible supersonic plasma jets but was limited to a pressure range down to 200 mbar $[6,7]$. Recently, new interpretations of enthalpy measurements have been developed in order to deduce the free stream jet properties from the stagnation parameter behind the shock in front of the probe $[8,9]$ but the departure from LTE and the low gas sampling rate make the enthalpy measurements impossible at lower pressures.

In parallel to the experimental research, numerous simulations have been developed, in particular, two-temperature models for low pressure plasma jets, but they lack experimental validation $[10,11]$. On the other hand, plasma jets expanding at pressures below 1 mbar have been extensively investigated both numerically and experimentally $[12,13]$.

At present, the investigated pressure range to characterize plasma jets is either close to atmospheric pressure or at very low pressure. All the above-mentioned limitations show the need to develop a specific diagnostic tool for DC plasma jets applicable in the pressure range from 2 to 200 mbar and which is independent of LTE. Electrostatic probes could be one diagnostic of choice. They are widely applied in low density plasmas, such as glow discharges, to measure the electron temperature and density. They are rarely used in thermal plasmas because of the high heat-load and highly collisional regime. However, measurements of electron density and temperature have 
been performed in transferred arcs and in atmospheric pressure plasma jets [14, 15, 16]. Since the seventies, electrostatic probes have been used to study the ionosphere by measuring the electron density, electron temperature and in particular, flow Mach numbers in plasma-space vehicle interaction using double crossed Langmuir probes called Mach probes. For this purpose several theories were developed taking into account the effects of a supersonic flow on the current collection such as wake effects or sheath deformation [17, 18]. A compilation of the different cases is presented by Tan [19]. Measurements with double probes and Mach probes were performed for nitrogen and argon plasma jets but at very low pressures $(<0.8$ mbar) in parallel with spectroscopic measurements $[20,21,22]$. These plasma jets exhibit a strong expansion with the creation of a normal shock and subsequent subsonic flow. In this case, the Langmuir probes allow a local characterization of the plasma jet structure.

In our case, to characterize the jets expanding at low pressure, two dedicated measurement techniques based on electrical probes have been developed and applied: the double Langmuir probe and the Mach probe. The classical interpretation of the current-voltage characteristic of a double probe can be used provided that the sheath is thin and collisionless, which is the case in most of the conditions between 2 and 80 mbar as will be shown below.

In this paper, the validity of these techniques is presented with a discussion in terms of mean free paths, Knudsen number and collisionality showing the domain of applicability of the electrostatic probe measurements. We present radial and axial profiles of Mach number, electron density and electron temperature for different chamber pressures between 2 and 40 mbar, different torch currents and argon gas flows. The axial measurement positions correspond to the middle of the expansion and compression zones encountered in these supersonic plasma jets. Complete mappings of Mach number, electron temperature and density of the jet were performed for under-expanded jets.

\section{Experimental set-up}

The plasma jet is generated by a Sulzer Metco F4-VB gun with a conical nozzle of $6 / 12 \mathrm{~mm}$ inner/exit diameter. It is mounted on a 2-axis (radial and axial) displacement system inside a $2 \mathrm{~m}^{3}$ vacuum vessel (figure 1). A 3-stage pumping system equipped with pressure feedback controlled throttle valves regulates the chamber pressure in the range of 2 to 500 mbar. Because of the high heat load and high collisionality of the plasma jet at high pressure, only the pressure range between 2 and 40 mbar is investigated in this study. The two main operation conditions used in this work are 40 SLPM of argon flow with a 400 A current and 60 SLPM argon flow with a 600 A current giving a net power in the plasma jet of $7 \mathrm{~kW}$ and $12 \mathrm{~kW}$ respectively.

The double Langmuir probe is made of two parallel tungsten wires $0.15 \mathrm{~mm}$ diameter protruding $1.5 \mathrm{~mm}$ from a thin ceramic tube. The two wires are $1 \mathrm{~mm}$ apart and the probe is quickly swept radially through the plasma jet by a rotating arm with calibrated position to obtain radial profiles. The current is calculated by measuring the 


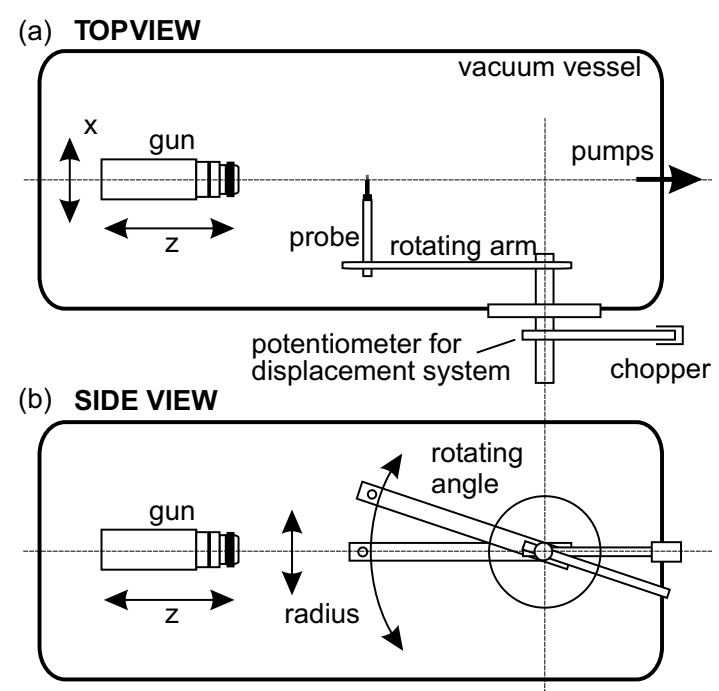

Figure 1. Schematic view of the experimental arrangement

voltage difference through a resistor $(4 \Omega)$ placed in the electrical circuit between the two probes. The current circulating between the electrically floating probes once immersed in the plasma jet is measured as a function of the applied voltage difference. The time of immersion has been optimized as a compromise between a fast sweep to avoid probe overheating or melting and a slow sweep to allow filtering out of the torch fluctuations (300 Hz produced by the power supply, high frequencies due to the arc fluctuations and flow turbulence). The optimal radial sweep time is found to be between 20 to $30 \mathrm{~ms}$.

The Mach probe construction is similar to the double probe, except that the two probes are mounted with their axes perpendicular to each other. Therefore one probe is parallel to the plasma jet flow, the other being perpendicular [23]. Each probe is polarized at the same potential in the ion saturation current region of the Langmuir characteristic. The ratio of the currents collected by the two probes allows determining the Mach number of the plasma flow as explained below. Before the measurements, the probes were positioned so that each one was perpendicular to the plasma flow, ensuring that the two probes have an equal current collection area. The optimal length of the probes with respect to their diameter has been found to be $1.5 \mathrm{~mm}(l / d \geqslant 10)$ so that the probes only collect ions through the lateral sheath surface [24]. The rotating arm is long enough to minimize the angle between the parallel probe and the jet axis when the probe is at the plasma jet fringes (see figure 1 (b)). At lower pressure, where the plasma jet diameter is the largest, the maximum angle is about $5^{\circ}$.

The data acquisition system which is the same for the two types of probes is a LeCroy ${ }^{\circledR}$ digital oscilloscope $(500 \mathrm{MHz})$. For the double probe, its 4 channels record simultaneously, for each radial sweep, the probe current, the applied voltage, the voltage corresponding to the radial position of the probe and a trigger signal giving the absolute position of the probe. Since the applied voltage is constant for the Mach probe, the first 2 channels record the currents flowing in each probe, the position measurements being 
the same as for the double probe.

A fast 12 bit CCD camera (SensiCam Fast Shutter from PCO [25]), equipped with an 18-108 $\mathrm{mm}$ zoom lens and a neutral density filter, is used to acquire images of the total plasma jet emission for different torch parameters and chamber pressures allowing a phenomenological description of the plasma jet flow topology. The camera is also used to monitor the alignment of the electrical probe on the jet axis while performing the radial sweeps (for this purpose it is positioned perpendicular to the probe rotating shaft and the jet axis).

\section{Probe theory and data analysis}

\subsection{Double probe}

The use of double probes is more appropriate than single probes in plasmas which have no reference electrode such as flowing plasma jets. The double probe system forms a floating closed circuit, so that no net current drain is taken from the plasma. Moreover the total current drawn by the probes will never be higher than the ion saturation current. The probe current as a function of the applied voltage difference, called the double probe characteristic (DPC), is used to determine the electron temperature and electron density. The DPC can be expressed analytically using the relation presented by Chen [26],

$$
I=I_{\text {sat }} \tanh \left(e U / 2 k T_{e}\right)
$$

where $I$ is the current flowing between the probes, $I_{\text {sat }}$ the ion saturation current, $T_{e}$ the electron temperature in Kelvin, $k$ the Boltzmann constant, $e$ the electron charge and $U$ the voltage difference. By differentiating this relation with respect to the voltage, the electron temperature can be determined from the slope of the DPC at the voltage $\mathrm{U}=0$ :

$$
\frac{k T_{e}}{e}=\frac{I_{s a t}}{\left.2 \frac{d I}{d U}\right|_{U=0}} .
$$

The electron density can be determined by measuring the ion saturation current drawn by the probes (which is equal to the ion current across the sheath surface). Assuming a collisionless sheath and using the Bohm criterion at the edge of the sheath, the electron density can be calculated with the relation presented by Hutchinson [27]

$$
I_{\text {sat }}=\exp \left(-\frac{1}{2}\right) A_{s} n_{e}\left(\frac{T_{e}}{m_{i}}\right)^{\frac{1}{2}}
$$

where $A_{s}$ is the area of the sheath surface, $m_{i}$ the ion mass and $n_{e}$ the electron density. If the sheath is thin, which is the case here, the sheath area can be approximated by the surface area of the probe. The value of the current at the intersection between the ion saturation branch and the slope of the DPC at $U=0$ is used as the value of the ion saturation current (see figure 2(b)) [28]. 
Applying a periodically ramping voltage signal is not appropriate when performing fast radial sweeps of the probe. Therefore, to keep a good spatial resolution, a static potential is applied for one sweep of the probe in the plasma jet to obtain a radial profile of the current. The voltage is then incremented from -20 to $20 \mathrm{~V}$, for each radial sweep, to obtain the different current radial profiles like shown in figure 2 (a). The double probe characteristic in figure 2 (b) is built point by point for a specific radial distance which is marked by a vertical line in figure 2 (a). This means that about 20 measurements are necessary to reconstruct a double probe characteristic. With this method, any position in the plasma jet radius can be investigated with no loss of the spatial resolution. The data analysis is made with the commercial software MatLab ${ }^{\circledR}$, which allows to merge the data collected by the digital scope. As the measured current profiles show from the natural torch fluctuations a numerical Butterworth filter is applied to the raw signal before analysis.
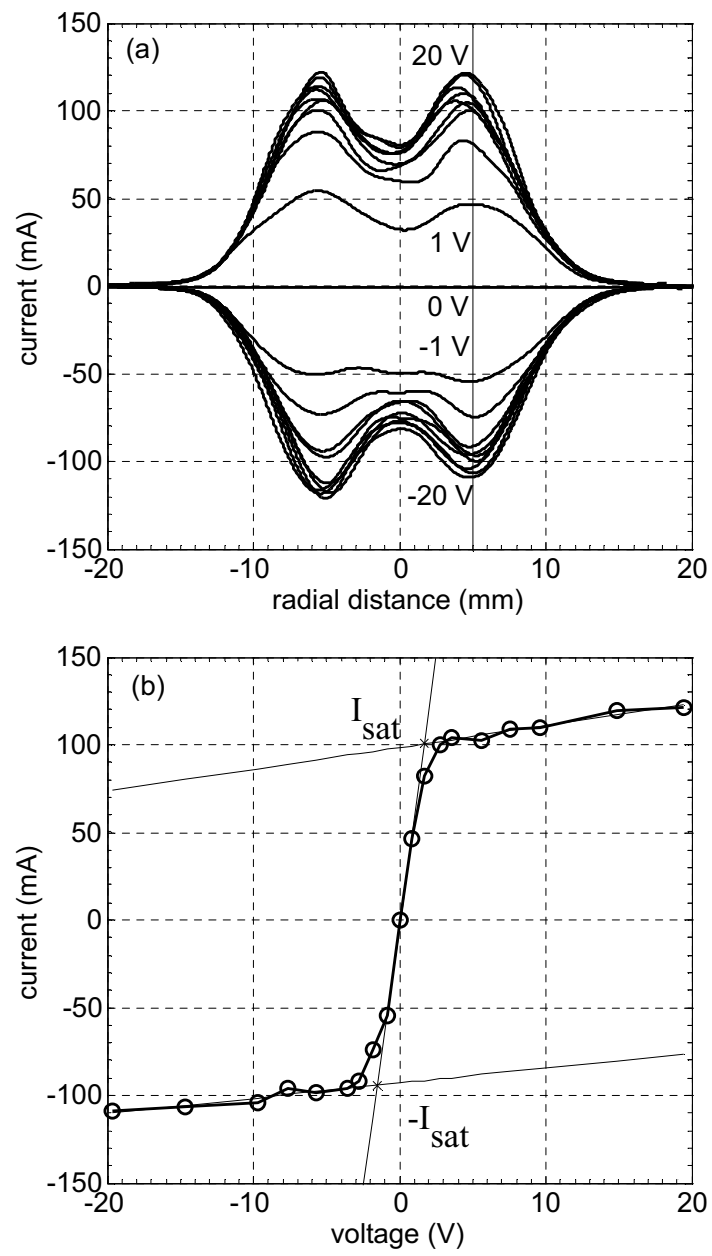

Figure 2. Filtered current radial profiles at various voltages from -20 to $20 \mathrm{~V}$ (a). The corresponding DPC built for the radial position $\mathrm{r}=10 \mathrm{~mm}$ (b). Plasma conditions: 400 A current, 40 SLPM Ar, 10 mbar chamber pressure. 


\subsection{Mach probe}

The ratio of the currents collected by the two probes $\left(I_{\perp} / I_{\|}\right)$is used to calculate the Mach number at different locations in the plasma jet. In this case the theory presented by Murphree et al. is used [23]. The two probes are polarized at the same potential in the ion saturation current region of the Langmuir characteristic. If the sheath is collisionless and smaller than the probe radius, which applies in our case, and if the current collection areas are equal for the two probes, the following relation can be used:

$$
\frac{I_{\perp}}{I_{\|}}=\frac{2}{\pi^{1 / 2}} e^{-\left(v / c_{m}\right)^{2}} \sum_{n=0}^{\infty}\left[\frac{\left(v / c_{m}\right)^{n}}{n !}\right]^{2} \Gamma\left(n+\frac{3}{2}\right)
$$

Where $\Gamma(z)$ is the gamma function.

The ion Mach number can be determined, which is given by $v / c_{m}, v$ being the ion flow velocity and $c_{m}$ the ion sound velocity. In this case, it is assumed that the ion Mach number is equal to the neutral Mach number, which gives the flow Mach number.

If $v>c_{m}$ (supersonic flow), a correction factor to the current collection area of the perpendicular probe has to be introduced, which accounts for a wake effect [23, 29]. In our case, the current flowing in the perpendicular probe is always smaller than the parallel one, at any location in the plasma jet. This shows that the collection area of the perpendicular probe is reduced by the wake effect. For a supersonic flow, the ion drift velocity is high enough to avoid collection of the thermal ions that enter the sheath downstream of the probe mid-plane. Therefore we assume that only the half of the probe cylinder surface facing the plasma jet collects the ion current and we correct the measured perpendicular current accordingly.

\subsection{Discussion on the validity of the theory}

The domain of validity of the theories applied to electrostatic probes can be evaluated depending on the value of the Debye length $\lambda_{D}$, the probe dimension $R$ (typically the probe radius), the mean free path $\lambda_{\alpha \beta}$ of the particle $\alpha$ through a field of particles $\beta$ and the Knudsen number $K n$ of the probe. $K n$ is defined by the ratio of the mean free path $\lambda_{\alpha \beta}$ of the considered species and the probe dimension $R, K n=\lambda_{\alpha \beta} / R$. The classical Langmuir probe theory is valid if $\lambda_{\alpha \beta} \gg R \gg \lambda_{D}$, which corresponds to a thin and collisionless sheath. In order to verify this, the different conditions are considered separately.

The condition $R \gg \lambda_{D}$ corresponds to a thin sheath. The Debye length has been calculated assuming LTE for different electron temperatures between 0.5 and $1.5 \mathrm{eV}$, where the densities between $6 \times 10^{16} \mathrm{~m}^{-3}$ and $1 \times 10^{23} \mathrm{~m}^{-3}$ correspond to the working pressures between 2 and 100 mbar. $\lambda_{D}$ is in the range of 60 to $9000 \mathrm{~nm}$ and since the probe radius is $75 \mu \mathrm{m}$, the condition $R \gg \lambda_{D}$ is verified for the cases considered here.

The condition $\lambda_{\alpha \beta} \gg R(K n \gg 1)$, with $\lambda_{\alpha \beta}=\lambda_{i n}, \lambda_{e n}, \lambda_{e i}$, ensures that the classical Langmuir probe theory can be applied, in particular, $\lambda_{\alpha \beta} \gg \lambda_{D}$ corresponds to a non-collisional sheath. Furthermore, the condition $\lambda_{\alpha \beta} \gg R$, with $\lambda_{\alpha \beta}=\lambda_{n n}, \lambda_{i n}$ 
has to be satisfied to ensure that there is no formation of a shock in front of the probe tip. For this purpose, the different mean free paths for pressures between 2 and 80 mbar have been estimated. It has been found that $\lambda_{n n}$ is the smallest of the mean free paths. Therefore, to specify the domain of validity of the theory $\lambda=\lambda_{n n}$ is considered in this case. The condition $\lambda \gg R$ is verified if the temperature is higher than $0.7 \mathrm{eV}$ for pressures between 2 and 80 mbar. It should be noted that the mean free paths for pressures lower than 10 mbar are long, showing that collisions are less frequent throughout the entire length of the plasma jet. This is an indication that such plasma jets are in the transition regime discussed below. The combination of the two abovementioned conditions shows that $\lambda \gg \lambda_{D}$ is satisfied for all the temperatures from 0.5 to $1.5 \mathrm{eV}$ and pressure from 2 to $100 \mathrm{mbar}$, which means that the sheath is collisionless.

The absence of a shock in front of the probe implies that the measured values correspond to the free stream properties of the plasma jet. This is no longer the case at pressures higher than 40 mbar and for temperatures lower than $0.7 \mathrm{eV}$ where the mean free paths start to be of same dimension as the probe.

\section{Results}

In the first part of this section a qualitative description of the plasma jet flow phenomenology based on imaging is presented for different chamber pressures and torch parameters. The second part focuses on a quantitative characterization of the plasma jet, based on the double probe and Mach probe measurements.

\subsection{Plasma jet flow phenomenology}

Figure 3 shows images of the plasma jet total emission for different chamber pressures in the range of 10 to 80 mbar. For pressures above 30 mbar with the torch parameters given in figure 3, the jet is over-expanded and is characterized by the fact that the chamber pressure is higher than the nozzle exit pressure [9]. This results in the formation of oblique shocks at the edge of the nozzle exit, converging to the jet axis. These shocks turn the flow towards the axis which leads to a local compression. The local static pressure in the compression zone is higher than the surrounding pressure leading to an expansion of the jet downstream. The succession of compression/expansion zones repeats until the local static pressure is in equilibrium with the surrounding pressure, due to viscosity and turbulence [8].

For pressures below 30 mbar the jet is under-expanded and is characterized by an expansion with a larger diameter than the exit diameter of the nozzle. The edges of the first expansion zone are brighter than on axis because of the reflection of the expanding flow by the cold dense surrounding gas. This leads to the creation of so-called barrel shocks, visible on the plasma jet images. Once this reflection is passed, the plasma is compressed on the axis where part of the kinetic energy is converted into thermal energy. In this area, the local density and temperature increase which leads to an increase of the 


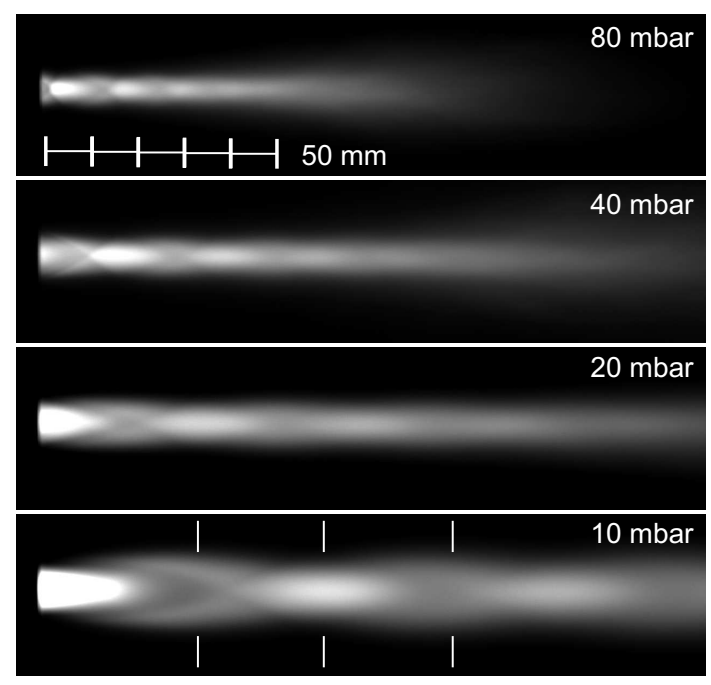

Figure 3. Images of the plasma jet at different chamber pressures. Current: 400 A, gas flow: 40 SLMP Ar. In this case the "design pressure" is 30 mbar

light emission. After this compression, the jet expands again because the local static pressure in the compression zone is higher than the surrounding pressure. This process of compression and expansion continues until the static pressure in the jet reaches the chamber pressure.

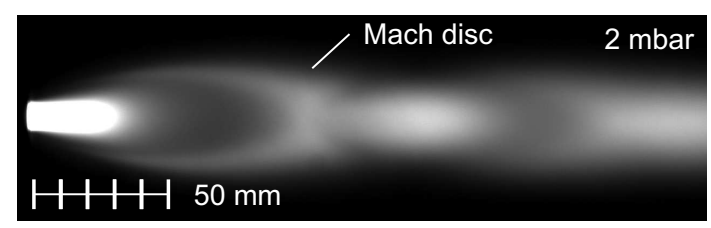

Figure 4. Image of the plasma jet showing the Mach disc for pressures below 6 mbar. Chamber pressure: 2 mbar, 400 A torch current, 40 SLPM Ar flow

In figure 4, for pressures below 6 mbar the jet is highly under-expanded which leads to a Mach reflection (Mach disc) downstream of the first expansion cell. This can bring the plasma jet to subsonic velocities downstream of this region with an increase of the local temperature and pressure.

At these low pressures, the jet dimensions are increased and the global light emission is strongly reduced. The plasma jet appears more diffuse due to flow rarefaction effects [12]. Here the plasma jet cannot be described by classical gas dynamics because the flow is no longer in a continuum regime. Muntz et al. [12] have defined a rarefaction parameter for jet exhausts to characterize the type of flow as a function of the exit pressure, chamber pressure, temperature and exit diameter. In our case, the transition regime occurs for pressures between 2 and 15 mbar and is characterized by the fact that the shocks become thicker and diffuse [9]. If the pressure were reduced further, the shock structure would disappear and the plasma jet would enter the scattering regime [12]. But in our case, this would occur only for pressures lower than 2 mbar, which are not 
studied in this paper. Plasma jets, operated at very low chamber pressures $(<0.2 \mathrm{mbar})$, generated by cascaded-arc torches, have been extensively studied and modeled [13, and references therein]. Such plasma jets are characterized by a strong expansion with an increase of the velocity and the presence of a subsequent normal shock and the absence of the periodic compression/expansion structure. The flow also enters a so-called frozen state where the plasma composition remains unchanged along the flow.

\subsection{Electrostatic probe measurements in an under-expanded jet at 10 mbar}

In this paragraph, probe measurements made at a chamber pressure of 10 mbar are presented which show the typical trends of under-expanded jets for the pressure range of 6 to 40 mbar. The torch parameters used here are a current of $400 \mathrm{~A}$ and a gas flow of 30 SLPM Ar. Figures 5 and 6 show radial profiles of Mach number, electron density and temperature at axial positions corresponding to the middle of successive expansion and compression cells which are indicated by vertical markers in figure 3. These positions correspond to the local extrema of the axial light emission profile.

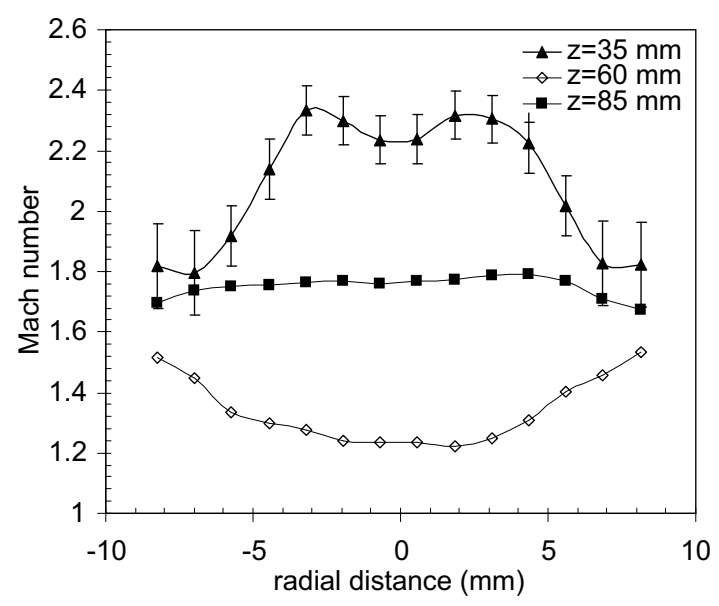

Figure 5. Radial profiles of the Mach number at different axial locations at 10 mbar, 400 A, 40 SLPM Ar

The Mach number radial profile (figure 5) in the first expansion zone at $\mathrm{z}=35 \mathrm{~mm}$ is broad and slightly hollow on axis. The two bumps correspond to the inner part $(\mathrm{r}=3.5 \mathrm{~mm})$ of the visible barrel shocks at the edge of the plasma jet where the flow reaches Mach 2.4. The flow velocity is then reduced radially to Mach 1.8 which is still supersonic because the shocks at the plasma fringe are oblique. In the compression zone $(\mathrm{z}=60 \mathrm{~mm})$, the Mach number radial profile presents a minimum on the jet axis. Its value strongly drops down to Mach 1.2 close to the subsonic transition but the flow remains supersonic, which implies that there is no Mach reflection. In this area, where the local static pressure is higher than the surrounding pressure, the jet starts to expand again, but due to the viscosity and turbulence, the plasma jet does not reach the speed of the first expansion zone and the profile is rather flat at Mach 1.7 in the second expansion cell $(\mathrm{z}=85 \mathrm{~mm})$. 

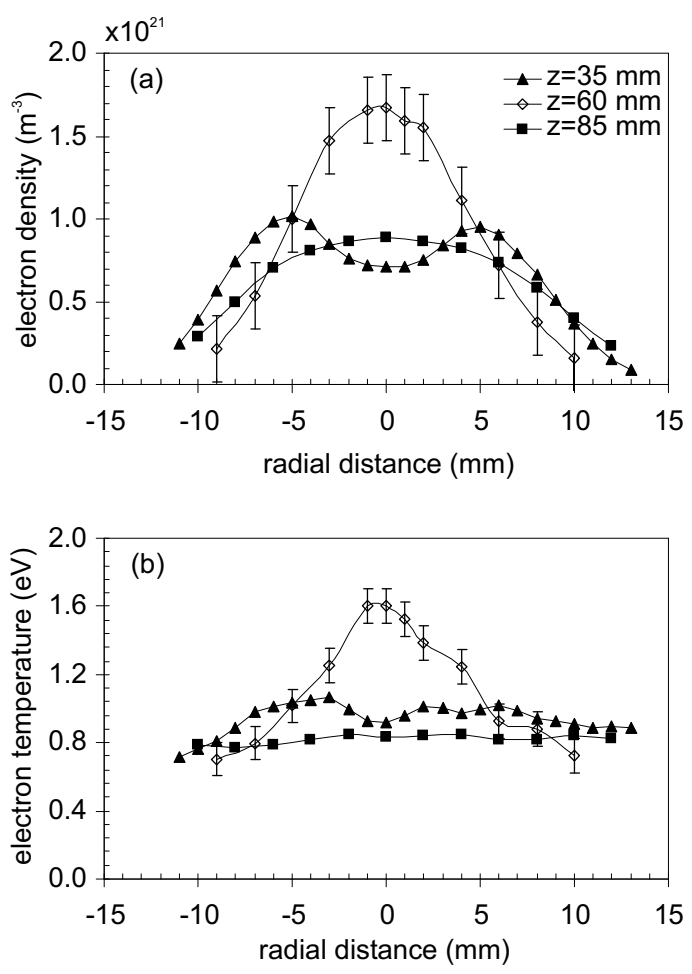

Figure 6. Radial profiles of the electron density (a) and electron temperature (b) at 10 mbar, 400 A current and 40 SLPM Ar flow

The radial profiles of the electron density shown in figure 6 (a) reproduce the structure of the jet topology characterized by the plasma jet light emission. The electron density is broad and hollow in the first expansion zone, so is the radial intensity profile. At the maximum expansion region the electron density is at its lowest value of $7 \times 10^{20} \mathrm{~m}^{-3}$ on axis. It has to be noted that the maximum of the electron density is located beyond the barrel shocks at $\mathrm{r}=5 \mathrm{~mm}$ whereas the Mach number already drops on the inner side of the barrel shocks at $r=3 \mathrm{~mm}$. Downstream, in the compression zone $(\mathrm{z}=60 \mathrm{~mm})$, the electron density radial profile presents a peak on axis $\left(1.7 \times 10^{21} \mathrm{~m}^{-3}\right)$ and is less broad than in the expansion zone because the jet diameter decreases due to the oblique shock waves that turns the flow towards the axis. In the second expansion zone the electron density is broad and does not present the two maxima at the edge like in the first expansion zone, because there are no barrel shocks there. The value on axis is about the same for the two expansion zones at $7-8 \times 10^{20} \mathrm{~m}^{-3}$. On the other hand the electron temperature radial profiles (figure $6(\mathrm{~b})$ ) show a rather flat profile in both expansion zones at values between 0.8 and $1 \mathrm{eV}$. However, it peaks in the compression zone where the axial value $(1.6 \mathrm{eV})$ is two times higher than on the plasma edge. There is a substantial heating of the electrons in the compression zone.

Figure 7 shows axial profiles of the Mach number, electron density and temperature and a direct comparison with the axial light emission of the plasma jet for the same conditions as for figure 5 and 6 . The Mach number clearly reaches its maximum when 

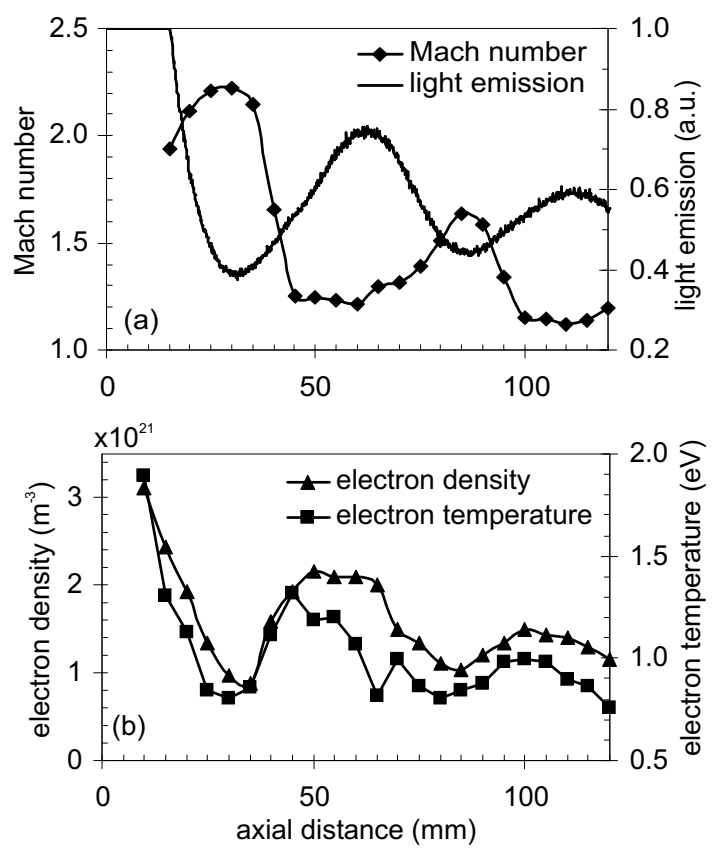

Figure 7. Axial profiles of the light emission and Mach number (a), and of the electron density and temperature (b) at 10 mbar, torch parameters: 400 A, 40 SLPM Ar

the jet is at its maximum expansion which corresponds to the region where the light emission is the weakest. Because of this expansion the axial electron density and the electron temperature also reach their minimum at $\mathrm{z}=30 \mathrm{~mm}$.

The oblique shock waves which allow the flow to change direction after the expansion reflect on the jet axis at an axial distance of about $\mathrm{z}=44 \mathrm{~mm}$. This region corresponds to a transition zone between the expansion and the compression zone (figure 3, 10 mbar). The Mach number strongly drops at this location and stays at a minimum as far as $\mathrm{z}=60 \mathrm{~mm}$ which corresponds to the maximum of light emission of the compression zone. The electron density and the electron temperature rise to their local maxima because the flow is strongly slowed down and compressed.

If we consider only the axial values, the electron density increases with a strong heating of the electrons from $0.8 \mathrm{eV}$ to $1.6 \mathrm{eV}$, which is evidenced by the axial profile in figure 7 . The plasma jet is strongly compressed and slowed down at the same time with a decrease of the Mach number from 2.4 to 1.2. Therefore a substantial fraction of the kinetic energy is converted into thermal energy. Beyond that point, the flow velocity already starts to increase till it reaches its maximum in the second expansion zone but remains still lower than in the first expansion zone. Axially, the electron density and temperature follow the structure of the plasma jet emission. However the Mach number strongly drops already upstream of the bright visible compression zone, even though the maximum of the Mach number occurs where the light emission is the weakest. This shows that emission is not an accurate monitor of the flow properties whereas it reflects the electron density and temperature changes quite well.

On the other hand, if we consider only the radial expansion in the first cell, there 
is also an increase of the electron density at the fringe but only with a slight increase of the electron temperature from 0.9 to $1.1 \mathrm{eV}$ (figure 6) and a slight reduction of the Mach number. In this case, there is only a reflection of the plasma jet at the edge by the cold surrounding gas with little conversion of the kinetic energy into thermal energy, unlike what happens in the compression zone.

\subsection{Plasma jet at very low pressure}

A mapping of the Mach number is presented to describe the flow of highly underexpanded jets. Figure 8 shows a contour plot of the Mach number along with the corresponding image of the jet emission at a chamber pressure of 2 mbar and for the same torch parameters as above.

(a)
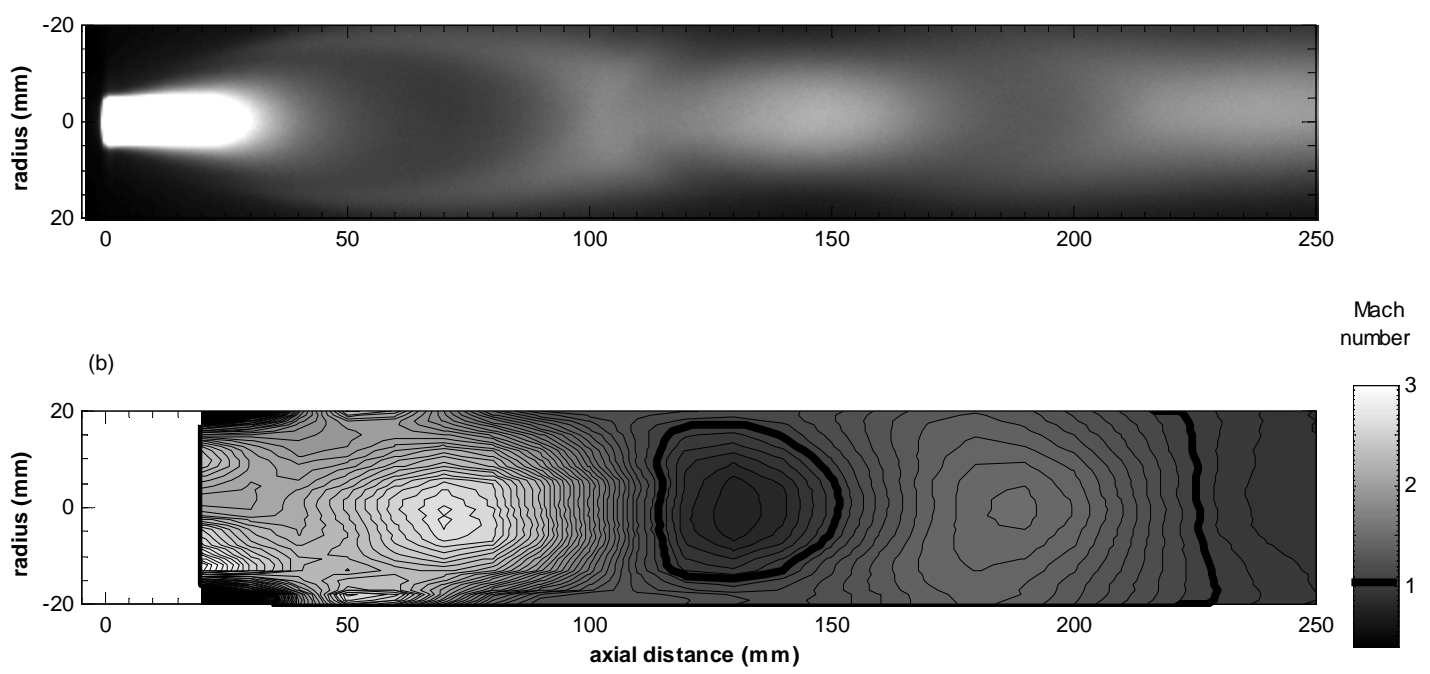

Figure 8. Total light emission (a) and Mach number iso-contours (b). Chamber pressure 2 mbar, 400 A current, 40 SLPM Ar flow

The Mach number reaches its maximum at about 2.8 in the first expansion cell at $\mathrm{z}=70 \mathrm{~mm}$. After this expansion, the iso-contour plots of the Mach number present straight narrow lines close to the axis which are perpendicular to the flow and correspond to a strong drop of the jet velocity. A corresponding increase of the light emission is observed around $\mathrm{z}=100 \mathrm{~mm}$. This area corresponds to the location where the oblique compression shock waves meet the Mach disc. In this case, the difference between the local static pressure and the surrounding pressure is so high that the oblique shocks are not sufficient to create an equilibrium between these pressures. Therefore a Mach reflection occurs between the oblique shocks [30]. The plasma jet becomes subsonic after crossing this normal shock. The subsonic region, delimited by the bold contour from $\mathrm{z}=120 \mathrm{~mm}$ to $\mathrm{z}=150 \mathrm{~mm}$ shown in figure 8 , corresponds to the first half of the bright 
compression cell which is centered at about $\mathrm{z}=150 \mathrm{~mm}$. The subsonic area does not cover the full plasma jet diameter, so that the plasma jet still remains supersonic in the surrounding of the compression cell. The delta shape of the Mach number iso-contours downstream of the subsonic area reveals the presence of oblique expansion waves. The Mach number reaches its second local maximum value in the second expansion zone, which is lower than in the first expansion zone. This zone present delta shaped isocontours, similar to the subsonic area, which in this case reveals the presence of oblique compression shock waves. The flow is slowed down at the plasma edge and is higher on the axis. Then a transition to a subsonic regime occurs throughout the whole diameter of the plasma jet which is marked by the bold line at $\mathrm{z}=225 \mathrm{~m}$, the flow remaining subsonic downstream.
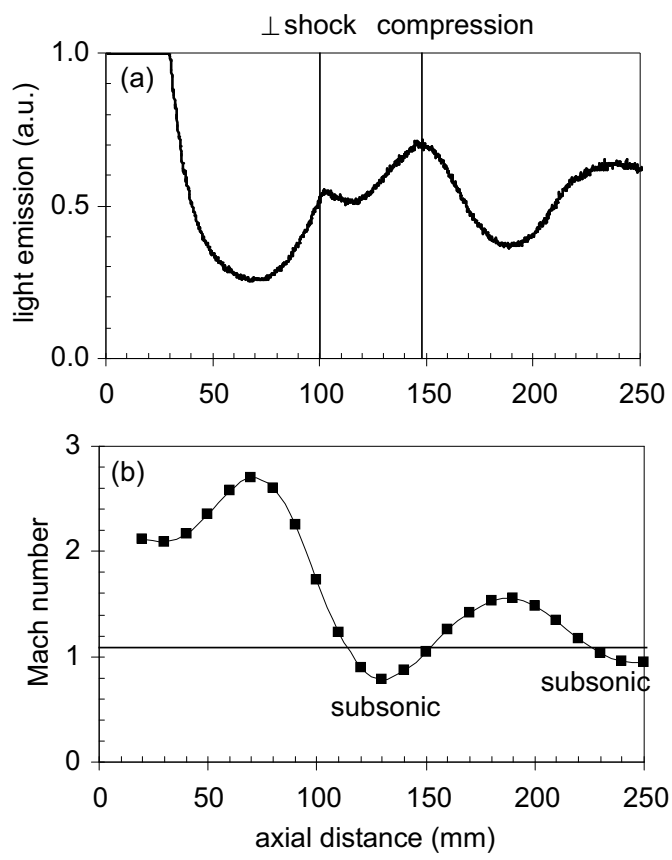

Figure 9. Axial profiles of the light emission and Mach number. Chamber pressure 2 mbar, 400 A current, 40 SLPM Ar flow

The Mach number and the light emission axial profiles for the same plasma conditions plotted in figure 9 confirm these effects. The Mach disc is evidenced by the first peak of the light emission at $\mathrm{z}=100 \mathrm{~mm}$ and the middle of the compression zone by the second peak at $\mathrm{z}=150 \mathrm{~mm}$. The drop of the Mach number is not as steep as in the 10 mbar case and extends over a region of $15 \mathrm{~mm}$ (4 mm at $10 \mathrm{mbar}$ ). The thickness of the shock would increase if the pressure was further reduced and the shock would even disappear at lower pressure if the jet was in the scattering regime. The subsonic region is clearly visible between $\mathrm{z}=115$ and $\mathrm{z}=145 \mathrm{~mm}$. At $\mathrm{z}=150 \mathrm{~mm}$, in the middle of the compression zone, the Mach number already starts to rise. 


\section{Summary and Conclusions}

Radial and axial profiles of parameters of a low pressure argon plasma jet, such as the Mach number, electron temperature and electron density were measured using double Langmuir probes and Mach probes. Unlike other diagnostics, the electrostatic probes do not rely on the assumption of local thermodynamic equilibrium, therefore they are an appropriate diagnostic to study low pressure plasma jets which are known to be nonLTE. Low pressure plasma jets also exhibit unconventional properties, such as large dimensions, supersonic flow and shock structures, which the double Langmuir probe and the Mach probe allow to explore locally.

The validity range of the classical Langmuir probe theory was verified with respect to the work pressure and the operation conditions by estimating the Debye length, the mean free paths and Knudsen numbers. It was shown that electrostatic probes are a reliable diagnostic for plasma jets covering a pressure range below 80 mbar. At higher pressures, the validity of the classical Langmuir probe theory is no longer satisfied and dedicated theories have to be used in order to make correct interpretation of the measurements. It was also shown that at pressures higher than 40 mbar, the mean free paths are reduced to the dimension of the probe so that a shock can be created in front of the probe, which would make the interpretation of the measurements of the plasma jet properties more difficult. It was shown that under-expanded plasma jets at lower pressure can reach Mach 2.8 with the creation of a Mach disc after the first expansion. Moreover, for highly under-expanded plasma jets, a subsonic region can be created downstream of the Mach disc when the torch current and the gas flow are low. It was also shown that the measured physical properties are consistent with the jet phenomenology such as the presence of expansion and compression zones, the effect of the pressure and the location of the shock waves.

In the pressure range studied, different regimes of the plasma jet exist: for pressures lower than 30 mbar, the plasma jets are under-expanded, above 30 mbar they are overexpanded. At pressures below 6 mbar, the plasma flow becomes rarefied, therefore classical gas dynamics can no longer be applied.

The results presented in this paper contribute to the understanding of the supersonic plasma jet behavior at low pressure and can be used to quantify the deviation from LTE. The extensive mapping of the measured physical properties of the jet will also serve as input for modelling.

\section{Acknowledgments}

This work was funded by the CTI Swiss Federal Research Projet No. KTI 4403.1KTS.

\section{References}

[1] Fauchais P, Vardelle A and Dussoubs B 1999 Journal of Thermal Spray Technology 101683

[2] Fauchais P and Vardelle A 2000 Plasma Phys. Control. Fusion 42 B365 
[3] Sember V, Gravelle D V and Boulos M I 2002 J. Phys. D: Appl. Phys. 351350

[4] Selezneva S E, Sember V, Gravelle D V and Boulos M I 2002 J. Phys. D: Appl. Phys. 351338

[5] Sabsabi M, Vacqui S, Gravelle D V and Boulos M I 1992 J. Phys. D: Appl. Phys. 25425

[6] Hollenstein M, Rhamane M and Boulos M I 1999 Heat and Mass Transfer Under Plasma Conditions 891377

[7] Fincke J R, Swank W D, Snider S C and Haggard D C 1993 Rev. Sci. Instrum. 643585

[8] Jodoin B, Gindrat M, Dorier J L, Hollenstein C, Loch M and Barbezat G 2002 in Proceedings of ITSC 2002 (International Thermal Spray Conference)

[9] Gindrat M, Dorier J L, Hollenstein C, Loch M, Refke A, Salito A and Barbezat G 2002 in Proceedings of ITSC 2002 pp. 459-464 (International Thermal Spray Conference)

[10] Han P and Chen X 2001 Thin Solid Films 290181

[11] Bartosiewicz Y, Proulx P and Mercadier Y 2002 J. Phys. D: Appl. Phys. 352139

[12] Muntz E P, Hamel B B and Maguire B L 1970 AIAA Journal 91651

[13] Burm K T A L, Goedheer W J and Schram D C 2001 J. Appl. Phys. 902162

[14] Leveroni E and Pfender E 1989 Rev. Sci. Instrum. 603744

[15] Gick A E F, Quigley M B C and Richards P H 1973 J. Phys. D: Appl. Phys. 61941

[16] Fanara C and Richardson I M 2001 J. Phys. D: Appl. Phys. 342715

[17] Jakubowski A K 1972 AIAA Journal 10988

[18] Kanal M 1963 J. Appl. Phys. 351697

[19] Tan W P S 1973 J. Phys D: Appl. Phys. 61188

[20] Robin L, Vervisch P and Cheron B G 1994 Phys. Plasmas 1444

[21] Tilley D L, Gallimore A D, Kelly A J and R G J 1994 Rev. Sci. Instrum. 65678

[22] Dudeck M, Poissant G, Rowe B R, Queffelec J L and Morlais M 1983 J. Phys. D: Appl. Phys. 16 995

[23] Johnson B H and Murphree D L 1969 AIAA Journal 72028

[24] Chung P M, Talbot L and Touryan K J 1975 Electric Probes in Stationary and Flowing Plasmas: Theory and Application (New York: Springer-Verlag)

[25] PCO AG 93309 Kellheim, Germany www.pco.de

[26] Chen F 1965 Plasma Diagnostic Techniques chapter 4 (New York: Academic Press) pp 113-200

[27] Hutchinson I H 1987 Principles of Plasma Diagnostics (Cambridge: Cambridge University Press)

[28] Schott L 1995 in Plasma Diagnostics chapter 11 (New York: AIP Press) pp 668-731

[29] Hall D F, Kemp R F and JR J M S 1964 AIAA Journal 21032

[30] Shapiro A H 1953 The Dynamics and Thermodynamics of Compressible Fluid Flow (John Wiley and Sons) p 425 